\title{
Produção animal e vegetal em pastagem nativa manejada sob diferentes ofertas de forragem por bovinos
}

\author{
Animal and vegetal production of a natural pasture under different forage allowances for cattle
}

\author{
Jean Carlos Mezzalira ${ }^{\mathrm{I}^{*}}$ Paulo César de Faccio Carvalho ${ }^{\mathrm{II}}$ Júlio Kuhn da Trindade ${ }^{\mathrm{I}}$ Carolina Bremm ${ }^{\mathrm{I}}$ \\ Lidiane Fonseca' ${ }^{\mathrm{I}}$ Márcio Fonseca do Amaral ${ }^{\mathrm{III}}$ Mônica Vizzotto Reffatti ${ }^{\mathrm{I}}$
}

\section{RESUMO}

Neste trabalho avaliou-se a influência de diferentes ofertas de forragem e suas combinações ao longo do ano sobre a dinâmica do crescimento da pastagem e o desempenho animal. $O$ experimento foi conduzido em área de pastagem natural com novilhas de sobreano mantidas em pastejo contínuo com taxa de lotação variável. Os tratamentos foram ofertas de forragem fixas ao longo do ano: 4; 8; 12 e $16 \mathrm{~kg}$ de matéria seca $100 \mathrm{~kg}$ de peso vivo-1 por dia e combinações de 8 na primavera e 12 no outono-inverno-verão (8-12\%); 12 na primavera e 8 no outono-inverno-verão (12-8\%); 16 na primavera e 12 no outono-inverno-verão (16-12\%), constituindo um delineamento experimental de blocos casualizados com duas repetições. A produção de forragem $e$ o ganho de peso animal foram medidos na estação de crescimento de 2007-2008. Os resultados comprovaram que o uso de oferta de forragem muito baixa, como 4\%, prejudica o desempenho animal individual e por área. O manejo combinado de ofertas $8-12 \%$ promoveu aumento de $35 \%$ no desempenho individual $\left(0,345 \mathrm{~kg} \mathrm{animal}^{-1}\right)$ aumento de $20 \%$ na produção por área (209kg ha ${ }^{-1}$ de $\left.\mathrm{PV}\right)$ em comparação ao manejo $12 \%$ ao longo do ano.

Palavras-chave: Bioma Pampa, pastagem nativa, sustentabilidade, produção de bovinos.

\section{ABSTRACT}

In this research it was evaluated the influence of different forage allowances and combinations of forage allowances along the year on the pasture accumulation dynamic and animal performance. The experiment was conducted in a natural pasture area with yearling beef heifers maintained in continuous grazing with variable stocking rate. The treatments utilized with fixed forage allowances during the year were 4; 8;

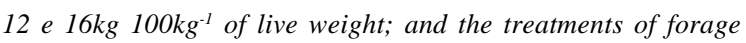
allowance combinations were 8 on Spring and 12 on AutumnWinter-Summer (8-12\%); 12 on Spring and 8 on AutumnWinter-Summer (12-8\%); 16 on Spring and 12 on AutumnWinter-Summer (16-12\%), constituting a experimental design in randomized blocks with two replicates of area. The primary and secondary productions were evaluated on the accumulation period of 2007-2008. The results proved that in situations of very low forage allowances, e.g. $4 \%$, the individual performance of animals and per area were prejudiced. Management of forage allowance combination of $8-12 \%$ promoted increase of $35 \%$ on the individual performance of animals $\left(0,345 \mathrm{~kg}_{\text {animal }}^{-1}\right)$, and of $20 \%$ on the production per area $\left(209 \mathrm{~kg} \mathrm{ha}^{-1}\right.$ of LW) when compared to a management of $12 \%$ over the year.
\end{abstract}

Key words: native pasture, Pampa Biome, sustainability, cattle production.

\section{INTRODUÇÃO}

Os ecossistemas pastoris correspondem a $40 \%$ da superfície terrestre, sem considerar a Groenlândia e a Antártida (NABINGER \& CARVALHO, 2009). O ecossistema pastagem nativa da região sul do Brasil ocupa 2,07\% do território nacional $\left(176.496 \mathrm{~km}^{2}\right)$ e comporta aproximadamente 13 milhões de bovinos e 5 milhões de ovinos (CARVALHO \& BATELLO, 2009). Essas formações campestres fazem parte do chamado Bioma Campos, que abrange desde o Uruguai, nordeste da Argentina, sul do Brasil e parte do Paraguai (PALLARÉS et al., 2005).

\footnotetext{
IPrograma de Pós-graduação em Zootecnia, Universidade Federal do Rio Grande do Sul (UFRGS), Avenida Bento Gonçalves, 7712, 91501-970, Porto Alegre, RS, Brasil. E-mail: mezzalirajc@gmail.com. *Autor para correspondência.

IIPrograma de Pós-graduação em Zootecnia, UFRGS, Porto Alegre, RS, Brasil.

IIEmpresa de Assistência Técnica e Extensão Rural (EMATER), Alegrete, RS, Brasil.
} 
Apesar da importância econômica e ambiental, a preservação e a utilização dessas pastagens naturais têm recebido pouca atenção por parte de governos (NABINGER \& CARVALHO, 2009). Sem melhorias significativas na produção da pecuária de corte, os índices produtivos são da ordem de $60-70 \mathrm{~kg}$ ha $^{-1}$ de PV por ano (CARVALHO et al., 2006). Por conta disso, frequentemente, e, erroneamente, este substrato é rotulado como pouco produtivo. Estes baixos índices zootécnicos e consequentemente econômicos desencadeiam problemas sociais, agrários e ambientais. Essa combinação de fatores faz com que a pastagem nativa perca, anualmente, cerca de $1000 \mathrm{~km}^{2}$, porção que é substituída por cultivos anuais, silvícolas e pastagens cultivadas (CORDEIRO \& HASENACK, 2009). Portanto, é necessária uma coordenação de esforços para compatibilizar a conservação e a produção desse patrimônio (CARVALHO \& BATELLO, 2009).

Pesquisas anteriores, neste mesmo protocolo, consolidaram diversos conhecimentos que garantem o aumento da produção animal e vegetal via ajuste da OF (MOOJEN, 1991; CORRÊA \& MARASCHIN, 1994; MOOJEN \& MARASCHIN, 2002; SOARES et al., 2005). Além do melhor desenvolvimento do aparelho reprodutivo de novilhas (SANTOS, 2007), a diversidade florística da vegetação (CARVALHO et al., 2003; CRUZ et al., 2010), a trajetória de dinâmica da vegetação (SOARES et al., 2005; HALFORD et al., 2010), entre outros conhecimentos, mantêm indicadores favoráveis, quando a OF é ajustada em níveis intermediários.

Trabalhou-se com a hipótese de que a imposição de distintas ofertas de forragem e a alteração da oferta na primavera cria estruturas de pasto distintas. Tais estruturas têm efeito sobre o desempenho de bovinos de corte em pastejo.

\section{MATERIAL E MÉTODOS}

O experimento foi conduzido entre abril de 2007 e março de 2008 em uma área de 60ha de pastagem natural pertencente à Estação Experimental Agronômica da Universidade Federal do Rio Grande do Sul, situada

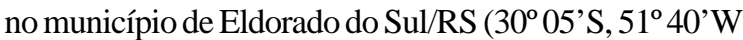
e $46 \mathrm{~m})$. O clima da região é subtropical úmido (Cfa) com verão quente. Apresenta precipitação média anual de 1440mm, distribuídos de forma desuniforme ao longo do ano, com valores máximos de 168,2mm em junho, e mínimo de 97,7mm em dezembro. As temperaturas médias mensais variam entre 14 e $25^{\circ} \mathrm{C}$.

Na área experimental, predominam dois tipos de solos: 1- Argissolo vermelho distrófico típico ou arênico, representado por solos profundos, bem drenados, textura franco-argilo-arenosa a francoargilosa, ácidos, pobres em fósforo, matéria orgânica e saturação de bases; 2- Plintossolo apresenta solos mediamente profundos, mal drenados, textura francoarenosa, ácidos, com baixos teores de matéria orgânica, fósforo e saturação de bases. A vegetação é caracterizada pela ocorrência de gramíneas, principalmente dos gêneros: Andropogon, Aristida, Axonopus, Bothriochloa, Briza, Bromus, Panicum, Paspalum e Stipa. Ocorrem, também, leguminosas dos gêneros Adesmia, Desmodium, Mimosa, Macroptilium, Trifolium, Vicia, dentre outros (BOLDRINI, 1997).

Os tratamentos foram constituídos por níveis de oferta de forragem diária (OF; kg de MS $100 \mathrm{~kg}^{-1}$ de peso vivo $=\%$ do PV) e combinações de sequências de OF, quais sejam: 4; 8; 12 e 16\% do PV durante todo ano, $8 \%$ na primavera e $12 \%$ no verão, outono e inverno (8-12\%); 12\% na primavera e 8\% no verão, outono e inverno (12-8\%) e $16 \%$ na primavera e $12 \%$ no verão, outono e inverno (16-12\%). A área experimental está sob efeito dos tratamentos de ofertas fixas desde 1986. Já os tratamentos de ofertas variáveis (por exemplo, o tratamento 8-12\%, que era originalmente $8 \%$ ) passou a ser ajustado como $12 \%$ na primavera, a partir do ano 2000. O delineamento experimental utilizado foi o de blocos completos ao acaso, sendo o fator de blocagem o tipo de solo, com duas repetições (piquetes de área entre 3 e 5ha).

O método de pastejo utilizado foi o contínuo com taxa de lotação variável, ajustada a cada 28 dias, valendo-se da técnica put-and-take. No início do período experimental, em 27 de abril de 2007, a área encontravase em diferimento havia 72 dias. A OF de primavera foi ajustada para o período entre 08/10/07 e 16/01/08. As demais avaliações foram em: 28/04; 01/06; 30/06; 31/07; 06/09; 06/10; 11/11 e 17/12 em 2007 e 15/01; 18/02 e 26/03 em 2008. Totalizando 331 dias o período experimental.

A massa de forragem (kg MS ha-1) foi avaliada a cada 28 dias, aproximadamente, usando-se a técnica de "dupla amostragem”. O número de estimativas visuais foi de 50 por piquete (unidade experimental). Usou-se um quadrado metálico de $0,25 \mathrm{~m}^{2}$, com o qual se amostrou exclusivamente o estrato inferior do pasto, excluindo-se os pontos representados por touceiras (FONTOURAJr. et al., 2007). A média dos pontos foi estimada por uma equação de regressão do tipo $\hat{\mathrm{y}}=\mathrm{b} 0+\mathrm{b} 1$, gerada a partir de 54 outros pontos amostrados, cortados rente ao solo e estimados visualmente. As estimativas visuais foram realizadas por dois avaliadores por unidade experimental. $\mathrm{O}$ ajuste foi feito a partir da equação com $\mathrm{R}^{2}$ superior a 70 . A forragem cortada foi recolhida em sacos de papel, seca em estufa de ar forçado a $60^{\circ} \mathrm{C}$ por 72 horas e pesada em balança de precisão. 
A taxa de acúmulo de matéria seca (TAC; kg MS ha $^{-1} \mathrm{dia}^{-1}$ ) foi calculada a cada 28 dias, exclusivamente do estrato inferior do pasto, com o uso de quatro gaiolas de exclusão ao pastejo por unidade experimental. A altura do pasto foi medida com sward-stick em três pontos dentro de cada uma das 50 amostragens para estimativa indireta da massa de forragem. A amostragem obedeceu a um caminhamento sistemático. Para a altura do pasto, não foram consideradas as partes reprodutivas da planta, assim como material morto e touceiras não pastejadas. A frequência de touceiras (\%) foi obtida no mesmo procedimento da avaliação da altura do pasto. Ao se encontrar uma touceira, era anotada a sua observação sendo posteriormente calculada a sua frequência por regra de três entre os 50 pontos da unidade experimental e o número de pontos em que o quadro foi alocado em touceira.

Os animais utilizados foram novilhas mestiças, oriundas de cruzamento entre as raças Angus, Hereford e Nelore, com idade e peso médio inicial de 15 meses e $240 \pm 6 \mathrm{~kg}$, respectivamente. O peso médio inicial foi considerado como covariável. Foram utilizados quatro animais testers por unidade experimental e um número variável de animais reguladores ao longo do período experimental. Todos os animais eram pesados mensalmente, precedidos de jejum de sólidos e líquidos de 16 horas. Por ocasião das pesagens, os animais foram submetidos à avaliação do escore de condição corporal (ECC), de forma visual, utilizando-se de uma escala de notas entre 1 a 5 , sendo $1=$ muito magro e 5 = muito gordo (LOWMAN et al., 1973).

O ajuste mensal da taxa de lotação (kg PV $\mathrm{ha}^{-1}$ ) foi procedido com base na massa de forragem, na taxa de acúmulo de matéria seca, estimada para o período subsequente, e na OF pretendida para cada tratamento. O ganho médio diário (GMD; kg dia-1) correspondeu à diferença de peso individual dos animais testers entre o início e o fim de cada período, dividido pelo número de dias transcorridos. Já o ganho de peso vivo por hectare (GPV; $\mathrm{kg} \mathrm{ha}^{-1}$ ) foi calculado multiplicando-se o GMD dos animais testers pelo número de animais por hectare e novamente multiplicado pelo número de dias de cada período.

A oferta real de forragem (ORF) foi calculada pela seguinte fórmula: $\mathrm{ORF}=\{\{[(\mathrm{MFi}+\mathrm{MFf}) / 2] / \mathrm{n}+\mathrm{TA}\} /$ $\mathrm{CA}\}$, em que: $\mathrm{MFi}=$ massa de forragem inicial e $\mathrm{MFf}=$ =massa de forragem final do período $\left(\mathrm{kg} \mathrm{ha}^{-1} \mathrm{de}\right.$ MS); $n=$ número de dias do período; $\mathrm{TA}=$ representa a taxa de acúmulo diária ( $\mathrm{kg} \mathrm{ha}^{-1}$ de MS) e CA=carga animal (kg ha-1 de PV).

Os dados do GMD, ECC e peso vivo foram submetidos à análise de variância a 10\% de significância usando-se o procedimento LSMEANS e comparadas pelo teste t de Student. Foram realizadas análises de regressão até terceira ordem, conforme o modelo: $\mathrm{Y}_{\mathrm{ij}}=\mathrm{a}+\mathrm{bx}_{\mathrm{ij}}+\mathrm{cx}_{\mathrm{ij}}{ }^{2}+\mathrm{dx}_{\mathrm{ij}}{ }^{3}+\varepsilon_{\mathrm{ij}}$

Em que: $\mathrm{Y}_{\mathrm{ij}}=$ variável dependente; $\mathrm{a}=$ intercepto da regressão; $\mathrm{x}$ = variável independente; $\mathrm{b}$ = coeficiente linear de regressão da variável Y em relação à variável independente; c = coeficiente quadrático de regressão da variável Y relacionada à variável independente; $d$ = coeficiente cúbico de regressão da variável Y em relação à variável independente e $\varepsilon_{\mathrm{ij}}=$ erro aleatório residual.

Sempre que a equação de regressão foi significativa $(\mathrm{P}<0,10)$, optou-se por apresentar os resultados pela equação de maior coeficiente de determinação $\left(\mathrm{R}^{2}\right)$. Quando a equação de regressão apresentou a mesma tendência entre os tratamentos, foi realizada a comparação das equações através dos testes de paralelismo e de igualdade de interceptos e, havendo igualdade entre elas $(\mathrm{P}>0,10)$, uma nova equação foi gerada a partir do conjunto de dados dos tratamentos semelhantes. O modelo estatístico geral referente à análise das variáveis estudadas foi representado por: $\mathrm{Y}_{\mathrm{ijk}}=\mu+\beta_{\mathrm{i}}+\mathrm{T}_{\mathrm{j}}+(\beta \mathrm{T})_{\mathrm{ij}}+\gamma_{\mathrm{k}}+(\mathrm{T} \gamma)_{\mathrm{jk}}+\varepsilon_{\mathrm{ijk}}$.

Pelo modelo, $\mathrm{Y}_{\mathrm{ijk}}$ representa as variáveis respostas; $\mu$ é uma média inerente a todas as observações; $\beta_{\mathrm{i}}$ é o efeito do i - ésimo bloco; $\mathrm{T}_{\mathrm{j}}$ é o efeito do j - ésimo tratamento (oferta de forragem); $(\beta \mathrm{T})_{\mathrm{ij}}$ é o efeito aleatório devido à interação do i ésimo bloco com o j - ésimo tratamento (erro a); $\gamma_{\mathrm{k}}$ é o efeito do $\mathrm{k}$ - ésimo subperíodo observado (medida repetida no tempo); ( $\mathrm{T} \gamma)_{\mathrm{jk}}$ é o efeito da interação entre o j - ésimo tratamento com o k - ésimo subperíodo e $\varepsilon_{\mathrm{ijk}}$ corresponde ao erro aleatório, suposto independente e normalmente distribuído (erro b). Os dados foram analisados com o pacote estatístico SAS (2001).

\section{RESULTADOS E DISCUSSÃO}

A imposição das distintas OFs (Tabela 1) ao longo de 21 anos determinou condições distintas do dossel do pasto. A altura do pasto apresentou valores médios da ordem de 3,7 cm na OF 4\% e 8,5 cm na OF $16 \%$. A MF variou de $800 \mathrm{~kg} \mathrm{ha}^{-1}$ na OF $4 \%$ até $1700 \mathrm{~kg}$ $\mathrm{ha}^{-1}$ na OF $16-12 \%$. É evidente o contraste entre as OF $4 \%$ e $8 \%$, intervalo em que ocorre maior aumento na MF, já entre $8 \%$ e $16 \%$ este incremento ocorre com menor intensidade (Tabela 1).

No tratamento 8-12\%, mesmo apresentando OF real semelhante à OF $12 \%$, houve uma menor porcentagem de touceiras, decorrente do manejo sob a OF $8 \%$ na primavera. Este manejo estratégico proporciona porcentagem de touceiras semelhante ao tratamento $8 \%$, porcentagem também observada nesta 
Tabela 1 - Altura do pasto (cm); massa de forragem (MF; $\mathrm{kg} \mathrm{ha}^{-1}$ ); touceiras (\%); carga animal (CA; kg ha ${ }^{-1}$ de PV); taxa de acúmulo diário (TA; kg ha ${ }^{-1}$ de MS); produção de forragem (PMS; kg ha-1 de MS); produção animal (GPV; kg ha-1 ${ }^{-1}$ em pastagem nativa manejada sob distintas ofertas de forragem

\begin{tabular}{|c|c|c|c|c|c|c|c|c|}
\hline & \multicolumn{7}{|c|}{ 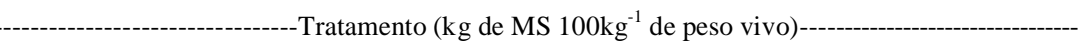 } & \multirow{2}{*}{$\mathrm{P}$} \\
\hline & $4 \%$ & $8 \%$ & $12 \%$ & $16 \%$ & $8-12 \%$ & $12-8 \%$ & $16-12 \%$ & \\
\hline Altura do pasto & $3,7 \mathrm{e}$ & $5,6 \mathrm{~d}$ & $7,9 \mathrm{ab}$ & $8,5 \mathrm{a}$ & $7,1 \mathrm{bc}$ & $6,7 \mathrm{c}$ & $8,7 \mathrm{a}$ & 0,0006 \\
\hline $\mathrm{MF}$ & $800 \mathrm{c}$ & 1257 b & $1398 \mathrm{ab}$ & $1530 \mathrm{ab}$ & $1428 \mathrm{ab}$ & $1268 \mathrm{~b}$ & 1696 a & 0,0277 \\
\hline Touceiras & $3,5 \mathrm{c}$ & $29,3 \mathrm{~b}$ & $38,2 \mathrm{ab}$ & 44,1 a & $26,8 \mathrm{~b}$ & $35,0 \mathrm{ab}$ & $41,5 \mathrm{a}$ & 0,0067 \\
\hline CA & $784 \mathrm{a}$ & $467 \mathrm{~b}$ & $303 \mathrm{~d}$ & $244 \mathrm{~d}$ & $420 \mathrm{~b}$ & $392 \mathrm{c}$ & $292 \mathrm{~d}$ & $<0,0001$ \\
\hline TA & $4,4 \mathrm{e}$ & $15,0 a b$ & $9,9 \mathrm{~cd}$ & $12,6 \mathrm{bc}$ & $17,2 \mathrm{a}$ & 6,9 de & $16,0 \mathrm{ab}$ & 0,0583 \\
\hline PMS & $1190 \mathrm{~d}$ & 3024 b & 2326 c & $2400 \mathrm{c}$ & 3867 a & 1499 d & $3026 \mathrm{~b}$ & 0,0059 \\
\hline GMD & 0,022 c & 0,197 b & $0,254 \mathrm{ab}$ & $0,246 a b$ & 0,345 a & $0,219 \mathrm{~b}$ & $0,272 a b$ & 0,0175 \\
\hline GPV & $150 \mathrm{~b}$ & $174 \mathrm{ab}$ & 127 bc & $95 \mathrm{c}$ & $209 \mathrm{a}$ & 135 bc & 132 bc & 0,0998 \\
\hline
\end{tabular}

*Valores seguidos de mesma letra, na linha, não diferem entre si pelo teste t a $10 \%$.

vegetação por NEVES et al. (2009) e permite aumento da produção animal em relação à OF fixa.

Observa-se que o tratamento $4 \%$ mantém carga animal média de aproximadamente $800 \mathrm{~kg} \mathrm{ha}^{-1}$. No entanto, o tratamento $8-12 \%$, mesmo sendo manejado a $8 \%$ apenas na primavera, e a $12 \%$ no restante do ano, suportou uma carga animal semelhante ao tratamento $8 \%$ e superior ao tratamento $12 \%$ (Tabela 1 ). Essa diminuição na OF na primavera, além dos benefícios na estrutura do pasto, permite aumento na capacidade de suporte da pastagem em relação ao tratamento $12 \%$ fixo ao longo do ano. Suportando o índice mínimo de lotação que considera um estabelecimento pecuário produtivo (0,8 UA=360 $\mathrm{kg} \mathrm{ha}^{-1}$ de PV) (CARVALHO \& BATELLO, 2009), este tratamento mantém média superior ao longo do ano.

A taxa média de acúmulo diário de forragem (Tabela 1) denota o efeito direto do pastejo sobre a produção do pasto. A mudança na OF de $8 \%$ na primavera para $12 \%$ nas demais estações do ano (8$12 \%$ ) se converte em incremento na TA da pastagem, resultando em maior produção de forragem anual. Os menores acúmulos foram observados no tratamento $4 \%$, que apresentou a menor média de altura do pasto. CORRÊA \& MARASCHIN (1994) observaram TA de 18 e $7 \mathrm{~kg} \mathrm{ha}^{-1}$ de MS nos tratamentos 16 e $4 \%$, respectivamente. PINTO et al. (2008) observaram taxas de 18 e $13 \mathrm{~kg} \mathrm{ha}^{-1}$ de MS para estas mesmas OF.

A estratégia de manejo 8-12\% condiciona uma estrutura do pasto de tal forma positiva ao processo de pastejo que o GMD médio do período experimental foi superior neste tratamento em relação ao tratamento $12 \%$ fixo ao longo do ano, mesmo tendo sido manejado por três meses sob OF 8\%. No período agosto-setembro, independentemente da OF, houve desempenho individual negativo (Tabela 2). Esse desempenho pode estar associado às baixas temperaturas ocorridas no segundo e terceiro decêndios do mês de julho, período em que se observou média das temperaturas mínimas de $3,1^{\circ} \mathrm{C}$ e vários dias com temperaturas negativas. Essa situação prejudicou a qualidade e estrutura do pasto a ponto de se refletir em perda de peso no período seguinte. Animais sob OF 4\%, caracterizada por proporcionar as menores alturas do pasto e MF, apresentaram o menor desempenho individual entre todas as OF.

No entanto, a passagem da OF 12 para a $16 \%$ traz uma grande influência sobre o processo de busca e colheita da forragem (MEZZALIRA et al., 2012), mesmo sendo um pequeno aumento da frequência de touceiras. O fato é que, aliado ao aumento da disponibilidade de forragem, ocorre um aumento na complexidade da estrutura da vegetação e, com isso, um aumento na dificuldade aos animais em explorá-la (LACA, 2008). Estas consequências negativas da estrutura do pasto se refletem no processo de colheita de forragem pelos animais e podem ser observadas, por exemplo, na igualdade de desempenho entre animais nas OF 12 e $16 \%$. O aumento na OF traz consigo aumentos na altura do pasto e na massa de forragem, mas estes não se refletem em aumento do desempenho animal.

Na média anual, o maior GMD foi registrado no tratamento $8-12 \%\left(0,345 \mathrm{~kg}_{\text {animal }}{ }^{-1}\right)$ mesmo sendo observados valores medianos, comparativamente aos demais tratamentos nos meses de primavera (setembro a dezembro), subperíodo em que foi manejado sob oferta de forragem $8 \%$ (Tabela 2), ou seja, esse tratamento apresentou média de $0,366 \mathrm{~kg} \mathrm{dia}^{-1}$ apenas superior ao tratamento $4 \%$, que proporcionou $0,184 \mathrm{~kg}$ $\mathrm{dia}^{-1}$. Este, aparente, prejuízo no desempenho 
Tabela 2 - Ganho médio diário (kg animal ${ }^{-1}$ ) de novilhas em pastagem nativa manejada sob distintas ofertas de forragem.

\begin{tabular}{|c|c|c|c|c|c|c|c|}
\hline & $4 \%$ & $8 \%$ & $12 \%$ & $16 \%$ & $8-12 \%$ & $12-8 \%$ & $16-12 \%$ \\
\hline Mai-jun & $0,719 * a b c$ & $0,444 \mathrm{~d}$ & $0,785 \mathrm{ab}$ & $0,510 \mathrm{~cd}$ & $0,934 \mathrm{a}$ & $0,541 \mathrm{bcd}$ & 0,705 abcd \\
\hline Jun-jul & $-0,384 \mathrm{c}$ & $0,056 \mathrm{ab}$ & $0,034 \mathrm{ab}$ & $-0,046 \mathrm{~b}$ & $0,272 \mathrm{a}$ & $-0,042 b$ & $0,069 \mathrm{ab}$ \\
\hline Jul-ago & $-0,236 \mathrm{c}$ & $-0,052$ bc & $0,160 \mathrm{ab}$ & $0,103 \mathrm{ab}$ & $0,262 \mathrm{a}$ & $-0,004$ abc & $0,109 \mathrm{ab}$ \\
\hline Ago-set & $-0,633 \mathrm{~b}$ & $-0,365 a b$ & $-0,246 \mathrm{a}$ & $-0,160 \mathrm{a}$ & $-0,160 \mathrm{a}$ & $-0,368 \mathrm{ab}$ & $-0,414 a b$ \\
\hline Set-out & $0,384 \mathrm{~b}$ & $0,628 \mathrm{ab}$ & $0,630 \mathrm{ab}$ & $0,579 \mathrm{ab}$ & 0,398 b & $0,519 \mathrm{~b}$ & 0,833 a \\
\hline Out-nov & $-0,054 b$ & $0,196 \mathrm{ab}$ & 0,365 a & 0,329 a & $0,165 \mathrm{ab}$ & $0,382 \mathrm{a}$ & 0,396 a \\
\hline Nov-dez & $0,221 \mathrm{~b}$ & 0,659 а & 0,643 a & 0,689 a & $0,536 \mathrm{a}$ & $0,744 \mathrm{a}$ & 0,679 а \\
\hline Dez-jan & $0,230 \mathrm{ab}$ & $0,034 \mathrm{abc}$ & $-0,103$ c & $0,211 \mathrm{ab}$ & $0,207 \mathrm{ab}$ & $-0,011$ bc & 0,266 a \\
\hline Jan-fev & 0,098 a & $-0,213 \mathrm{~b}$ & 0,167 a & $-0,103 a b$ & $0,167 \mathrm{a}$ & $-0,002 \mathrm{ab}$ & $-0,183 \mathrm{~b}$ \\
\hline Fev-mar & $-0,076 \mathrm{~d}$ & $0,607 \mathrm{ab}$ & $0,081 \mathrm{~cd}$ & 0,348 bc & 0,643 a & $0,393 \mathrm{ab}$ & 0,346 bc \\
\hline Média & 0,022 c & $0,197 \mathrm{~b}$ & $0,254 \mathrm{ab}$ & $0,246 \mathrm{ab}$ & 0,345 a & $0,219 \mathrm{~b}$ & $0,272 \mathrm{ab}$ \\
\hline
\end{tabular}

*Valores seguidos de mesma letra, na linha, não diferem entre si pelo teste t a $10 \%$.

individual, no tratamento 8-12\% quando manejado sob OF 8\%, é a estratégia fundamental para a manutenção da estrutura do pasto (menor frequência de touceiras e maior TA) e incremento do desempenho individual nos demais subperíodos do ano, em relação às OF 8 e 12\% e manutenção de maior carga animal.

A maior produção animal por hectare foi observada na OF 8-12\%, ratificando trabalhos anteriores (SOARES et al., 2005; PINTO et al., 2008) que observaram produções superiores dessa estratégia de manejo, em relação ao manejo com oferta $12 \%$ fixa ao longo do ano todo. O tratamento $4 \%$ apresentou elevado GPV anual em função de um elevado ganho médio diário de $0,719 \mathrm{~kg}_{\text {animal }}{ }^{-1}$ (Tabela 2) e uma carga animal de $1857 \mathrm{~kg} \mathrm{ha}^{-1}$ apresentados no primeiro período experimental, pós-diferimento de 72 dias. Essa capacidade de suporte, combinada ao alto GMD, proporcionou um GPV de $192 \mathrm{~kg} \mathrm{ha}^{-1}$ nos 36 dias de duração deste período, entre 27/04 e 01/06. Com uma análise detalhada da OF real (cálculo que leva em consideração o peso inicial e final dos animais), podese observar que nesse período a OF real foi de 2,2\%. Por conta da drástica mudança na estrutura do pasto, ocorrida no primeiro período, o desempenho individual na OF 4\% foi mais prejudicado no período seguinte.

O tratamento $8-12 \%$, por outro lado, combinou altas produções por área e a individual. Essa estratégia de manejo permite maior aproveitamento da forragem na primavera, via imposição de maior CA, coincidindo com o maior crescimento do pasto nesse período. Estratégia que permite maior desempenho individual nas demais estações do ano (Tabela 2).

A OF 4\% apresenta estrutura de pasto com valores de altura do pasto e massa de forragem inferior às metas de manejo recomendadas por SANTOS (2007) e SOARES et al. (2006). Isso conduz a uma determinante diferenciação do tratamento 4\%, quanto à evolução do peso vivo animal em relação aos demais ao longo do ano. Os animais do tratamento $4 \%$ tiveram um aumento de peso inicialmente por conta do diferimento prévio imposto ao pasto. Passado este período, houve uma acentuada perda de peso até o dia 132, estabilização até o dia 197 e, a partir disso, um discreto aumento até o final do período experimental. Entre os dias 263 e o dia 297 (Figura 1A), houve maior GMD, coincidindo com a primavera, período de maior crescimento do pasto.

Os demais tratamentos ajustaram-se no modelo linear de aumento do peso vivo médio ( $\mathrm{kg}$ animal $^{-1}$ ), aumentando em $0,216 \mathrm{~kg} \mathrm{dia}{ }^{-1}$. A alta variabilidade dos dados, principalmente entre os dias 234 e 297, expressa a alteração do peso vivo em função da alteração da OF que ocorreu na primavera. Mesmo assim, evidencia-se a distinção na evolução de peso vivo de animais submetidos à OF acima de $8 \%$ comparados a animais sob OF 4\%. NEVES et al. (2009), trabalhando no mesmo protocolo experimental, também observaram semelhança entre as ofertas acima de $8 \%$.

Considerando que a novilha atinge a maturidade sexual com aproximadamente $65 \%$ do PV da vaca adulta (450kg, em média), ou seja, 292,5kg (NRC, 1996), então apenas o tratamento $4 \%$ não permitiu aos animais atingirem tal peso ao longo do período experimental avaliado. Utilizando-se a equação de regressão gerada para os tratamentos de OF acima de $8 \%$, percebe-se que, partindo-se com novilhas pesando em torno de 240 e acréscimo diário de peso de $0,216 \mathrm{~kg}$ de PV (Figura 1A) tem-se a necessidade de 240 dias para que estas novilhas atinjam este peso mínimo. 


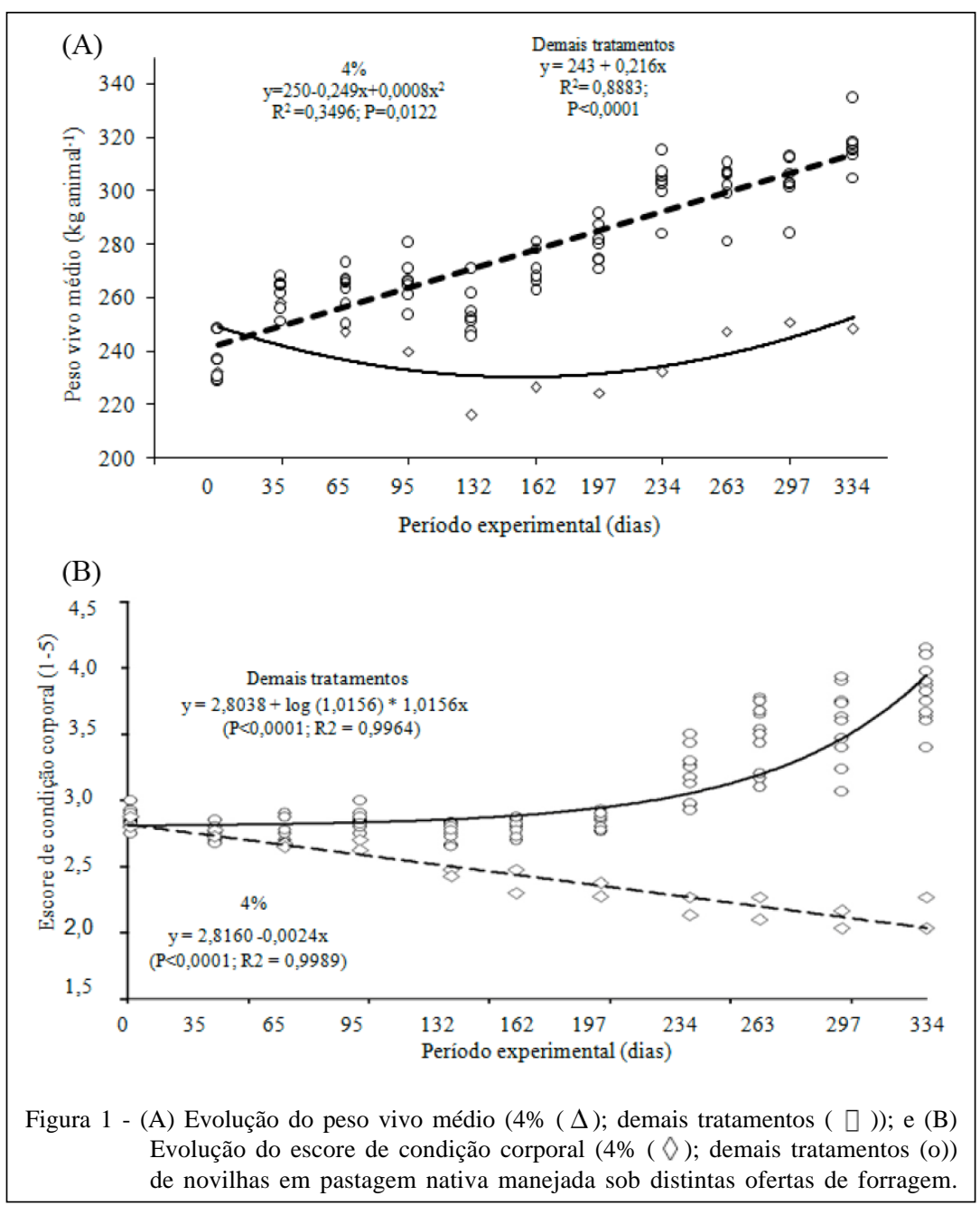

Os dados de condição corporal apresentados (Figura 1B) demonstram uma similaridade entre todos os tratamentos até o dia 197, a partir daí os animais submetidos à OF acima de $8 \%$ apresentaram um forte aumento até o final do período experimental no dia 334. No tratamento $4 \%$, porém, a condição corporal diminui linearmente ao longo de todo o período experimental. Ocorreu uma diminuição de 0,8 pontos, apresentando valor de 2 pontos de escore de condição corporal ao fim do período experimental. SANTOS (2007) também observaram valores próximos a 2,0 pontos de ECC neste tratamento. Estes autores consideraram estes valores de ECC como definitivos para inaptidão reprodutiva de novilhas mantidas nesta OF durante o segundo ano de recria. SANTOS et al. (2009) concluíram que a avaliação de ECC é uma prática eficaz para ser implementada como rotina no manejo do rebanho. Aliada ao peso vivo, a condição corporal é um bom indicador para decisões de manejo e aptidão reprodutiva.

\section{CONCLUSÃO}

Ofertas de forragem acima de $8 \%$ não apresentaram diferenças em relação à evolução do peso vivo médio e ECC. O tratamento de OF 8-12\% apresentou GMD e carga animal intermediários e alta produção animal por área. Apenas a oferta $4 \%$ é terminantemente indesejável na recria de novilhas de corte por proporcionar GMDs muito baixos e baixa produção do pasto.

\section{AGRADECIMENTO}

Ao Conselho Nacional de Desenvolvimento Científico e Tecnológico (CNPq), pela bolsa de estudo concedida ao primeiro autor. À Universidade Federal do Rio Grande do Sul (UFRGS), pela estrutura que garantiu a execução da pesquisa.

\section{REFERÊNCIAS}

BOLDRINI, I.I. Campos do Rio Grande do Sul: caracterização fisionômica e problemática ocupacional. Bol I Biociênc, v.56, p.1-39, 1997.

Ciência Rural, v.42, n.7, jul, 2012. 
CARVALHO, P.C.F.; BATELLO, C. Access to land, livestock production and ecosystem conservation in the Brazilian Campos biome: the natural grasslands dilemma. Livest Sci, v.120, p.158162, 2009. Disponível em: <http://www.sciencedirect.com/ science/article/pii/S1871141308001285>. Acesso em: $20 \mathrm{fev}$. 2009. doi: 10.1016/j.livsci.2008.04.012.

CARVALHO, P.C.F. et al. Produção animal no bioma campos sulinos. Rev Bras Zootec, v.35, p.156-202, 2006.

CARVALHO, P.C.F. et al. Herbage allowance and species diversity in native pastures. In: INTERNATIONAL RANGELAND CONGRESS, 7, 2003, Durban. Proceedings... Durban: New Millennium. Irene: Document Transformation Technologies, 2003, v.1, p.858-859.

CORDEIRO, J.L.P.; HASENACK, H. Cobertura vegetal atual do Rio Grande do Sul. In: PILLAR, V. de P. et al. (Eds.). Campos sulinos. Brasília: Ministério do meio ambiente, 2009. p.285-299.

CORRÊA, F.; MARASCHIN, G.E. Crescimento e desaparecimento de uma pastagem nativa sob diferentes níveis de oferta de forragem. Pesq Agropec Bras, v.29, p.16171623, 1994.

CRUZ, P. et al. Leaf traits as functional descriptors of the intensity of continuous grazing in native grasslands in the south of Brazil. Rangeland Ecol Manag, v.63, p.350-358, 2010. Disponível em: <http://www.bioone.org/doi/abs/10.2111/08-016.1>. Acesso em: 10 jun. 2010. doi: http://dx.doi.org/10.2111/08-016.1.

FONTOURA JR., J.A et al. Produção animal em pastagem nativa submetida ao controle de plantas indesejáveis e a intensidades de pastejo. Ciênc Rur, v.37, n.1, p.247-252, 2007. Disponível em: $<$ http://www.scielo.br/scielo.php?script=sci_arttext\&pid=S010384782007000100040\&lng=pt\&nrm=iso>. Acesso em: 10 set. 2008. doi: http://dx.doi.org/10.1590/S0103-84782007000100040.

LACA, E.A. Foraging in a heterogeneous environment: intake and diet selection. In: PRINS, H.H.T. et al. (Ed.). Resource ecology: spatial and temporal dynamics of foraging. Wageningen: UR Frontis Series, 2008. p.7-29.

LOWMAN, B.G. et al. Condition scoring beef cattle. Edinburgh: East of Scotland College of Agriculture, 1973. (Bulletin n.6).

MEZZALIRA, J.C. et al. The ingestive behaviour of cattle in large-scale and its application to pasture management in heterogeneous pastoral environments. J Agricult Sci Tech. v.2, n7A, 2012.

MOOJEN, E.L. Dinâmica e potencial produtivo de uma pastagem nativa do Rio Grande do Sul submetida a pressões de pastejo, épocas de diferimento e níveis de adubação. 1991. 172f. Tese (Doutorado em Zootecnia) Programa de Pós-graduação em Zootecnia, Faculdade de Agronomia, Universidade Federal do Rio Grande do Sul, Porto Alegre, RS.

MOOJEN, E.L.; MARASCHIN, G.E. Potencial produtivo de uma pastagem nativa do Rio Grande do Sul submetida a níveis de oferta de forragem. Ciênc Rur, v.32, p.127-132, 2002. Disponível em:
$<$ http://www.scielo.br/scielo.php?script=sci_arttext\&pid=S0103$84782002000100022 \& \operatorname{lng}=$ =t\&nrm=iso>. Acesso em: 03 jan. 2007. doi: http://dx.doi.org/10.1590/S0103-84782002000100022.

NABINGER, C.; CARVALHO, P.C.F. Ecofisiología de sistemas pastoriles: aplicaciones para su sustentabilidad. Agrocienc, v.13, n.3, p.18-27, 2009.

NEVES, F.P. et al. Estratégias de manejo da oferta de forragem para recria de novilhas em pastagem natural. Rev Bras Zootec, v.38, p.1532-1542, 2009. Disponível em: <http://www.scielo.br/ scielo.php ? script = sci_arttext \& pid=S 1516 35982009000800018\&lng=pt\&nrm=iso>. Acesso em: 22 dez. 2009. doi: http://dx.doi.org/10.1590/S1516-35982009000800018.

NATIONAL RESEARCH COUNCIL - NRC. Nutrient requirements of beef cattle. 7.ed.rev. Washington, D.C.: National Academy of Sciences, 1996. 242p.

PALLARÉS, O.R. et al. The South American Campos ecosystem. In: SUTTIE, J. et al. (Ed.). Grasslands of the world. Roma: FAO, 2005. p.171-219.

PINTO, C.E. et al. Produções primária e secundária de uma pastagem natural da Depressão Central do Rio Grande do Sul submetida a diversas ofertas de fitomassa aérea total. Rev Bras Zootec, v.37, p.1737-1741, 2008. Disponível em: <http:// www.scielo.br/scielo.php?script=sci_arttext \&pid=S151635982008001000004\&lng=pt\&nrm=iso>. Acesso em: $20 \mathrm{dez} .2008$. doi: http://dx.doi.org/10.1590/S1516-35982008001000004.

SANTOS, S.A. et al. Condição corporal, variação de peso e desempenho reprodutivo de vacas de cria em pastagem nativa no Pantanal. Rev Bras Zootec, v.38, p.354-360, 2009. Disponível em: <http:// www.scielo.br/scielo.php?script=sci_arttext\&pid=S151635982009000200019>. Acesso em: 10 fev. 2010. doi: http:// dx.doi.org/10.1590/S1516-35982009000200019.

SANTOS, D.T. Manipulação da oferta de forragem em pastagem natural: efeito sobre o ambiente de pastejo e 0 desenvolvimento de novilhas de corte. 2007. 259f. Tese (Doutorado em Zootecnia) - Universidade Federal do Rio Grande do Sul, Porto Alegre, RS.

SAS - Statistical analysis system. SAS/STAT user's guide: statistics. 4.ed. Version 8.2. Cary, 2001. V.2, 943p.

SOARES, A.B. et al. Produção animal e de forragem em pastagem nativa submetida a distintas ofertas de forragem. Ciênc Rur, v.35, p.1148-1153, 2005. Disponível em: <http://www.scielo.br/ scielo.php ? script = sci_art text \& pid=S 0103 84782005000500025\&lng=pt\&nrm=iso>. Acesso em: 10 jan. 2006. doi: http://dx.doi.org/10.1590/S0103-84782005000500025.

SOARES, A.B. et al. Efeitos de diferentes intensidades de pastejo em pastagem nativa melhorada sobre o desempenho animal. Rev Bras Zootec, v.35, p.75-83, 2006. Disponível em: <http:/ /www.scielo.br/scielo.php?script=sci_arttext\&pid=S151635982006000100009\&lng=pt\&nrm=iso>. Acesso em: 20 fev. 2006. doi: http://dx.doi.org/10.1590/S1516-35982006000100009. 